\title{
ANY QUESTIONS?
}

We publish below a selection of questions and answers of general interest.

\section{Iodides in Cataract}

Q.-Are iodides in the form of a local application or orally of any value in the treatment of cataract?

A.-There is no substantiated evidence that iodine in any form is of any value in the treatment of cataract.

\section{Collecting Clean Midstream Specimens}

Q.-How should a clean midstream specimen of urine suitable for bacteriology be obtained from (a) women attending a busy antenatal clinic; $(b)$ elderly women in hospital confined to bed; (c) infants and young children?

A.-Supervision of the collection of clean midstream urine in busy antenatal clinics is often impracticable, and the majority of unclean midstream specimens can be cultured by a screening method and reported negative. Therefore the recommended procedure is to hand the patient a wide-mouthed sterile jar or plastic container (minimum diameter 5 $\mathrm{cm}$.) and instruct her to catch the stream of urine while micturating into an ordinary water closet or one adapted for the purpose. ${ }^{1}$ Disposable plastic containers need not be sterilized if they are kept dust-free.

Positive cultures must be repeated even when an apparently pure growth is obtained, because coli of vaginal origin may be the only members of the vaginal flora capable of growing on the screening medium. For the repeat sample the patient is instructed to clean her vulva and vaginal orifice with soap and water and to dry thoroughly. She then takes the specimen by the same method, but is told on no account to allow the rim of the jar to touch her clothing or skin. Alternatively, a more elaborate method can be employed in which a nurse cleans the vulva and vaginal orifice with antiseptic and inserts a vaginal tampon. ${ }^{2}$ This should not be necessary provided culture and microscopy methods are aimed at recognizing accidental contamination from the vagina when present.

In the case of elderly bedridden women the following method is recommended. The patient sits on a bed-pan and the skin and external genitalia are cleaned with chlorhexidine $0.1 \%$ in $1 \%$ cetrimide added to an equal volume of warm tap-water. The skin is then dried very thoroughly. The patient is asked to pass water into the bedpan and the nurse collects the stream into a wide-mouthed jar. In order to facilitate collection a short glass funnel' or a sterile glass gravy boat $t^{5}$ have been recommended. Some patients have difficulty in passing water to order while reclining, and if the patient can sit on a bed-pan on a low stool at the bedside, or on a commode, a specimen may be more easily obtained.

In a proportion of elderly bedridden women a satisfactory specimen can be obtained direct from the bladder only by suprapubic puncture."

For infants and children too young to cooperate, a plastic collecting bag ("Chiron- seal," Great Ormond Street Hospital, Model B. Down Bros., Mayer and Phelps Ltd.) is employed. The large size, for children of from 3 months to 3 years, can also be cut to fit younger infants. The opening is cut to fit the child's vulva or penis in such a way that contamination from the anus is excluded. The vulva or penis is washed well with chlorhexidine in cetrimide solution (as above) and the skin dried thoroughly. The bag is then attached and removed as soon as possible after the child has micturated. Since external stimuli of any sort encourage micturition, the bag should be attached before other procedures-for example, weighing, blood sampling, etc.-are carried out. The urine is transferred to a sterile jar for rapid transport to the laboratory.

Older children should be cleaned and dried and then sat on a clean, deep chamberpot in the bottom of which is a wide-mouthed sterilized jar. If the child defaecates or fails to micturate at the first attempt the process, including the cleaning, must be repeated. Micturition may be encouraged by appropriate drinks given about 20 minutes before collection. In all cases culture within an hour of voiding or prompt refrigeration of the specimen is essential.

\section{REFBRENCBS} I Barnard, H. F., Brit. med. \%., 1965, 1, 1314. 1,395 .

Hutchings, M. T., Nursing Times, 1961, 57,

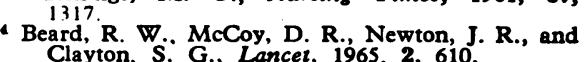
- Huxley Cowen, B. G. G., ibid., 1961, 2, 1460.

\section{Epilepsy and Television}

Q.-Television viewing may trigger off epileptic fits. Does the distance at which the child is viewing have any bearing on this?

A.-There is some clinical evidence to suggest that if the whole visual field is filled with the flickering light stimulus, photic epilepsy is more likely to be induced. This suggests that the nearer the child is to the screen the more likely it is for a fit to be triggered.

\section{Necrobiosis Lipoidica Diabeticorum}

Q.-Is there any specific treatment for necrobiosis lipoidica diabeticorum? Has this complaint anything to do with increase in weight?

A.-In many cases the lesions of necrobiosis lipoidica diabeticorum are insignifican and symptomless and call for no treatment. The lesions ultimately regress, usually leaving scars. Savitt ${ }^{1}$ reported a favourable response to injections of hydrocortisone into the lesions. Marten and Dulake ${ }^{2}$ used injections of $2 \mathrm{ml}$. of $25 \mathrm{mg} . / \mathrm{ml}$. hydrocortisone in 18 lesions in four patients, 17 of which showed improvement or complete resolution. However, instances of ulceration and cellulitis were attributed to the therapy.

The topical application of steroids may also be effective. ${ }^{3}$ Although the majority of patients with necrobiosis are diabetics who require insulin, it may appear some years before diabetes is manifest. ${ }^{5}$ The appearance of the lesion does not seem to have anything to do with an increase in weight.

\section{REFERENCES}

Savitt, L. E., Arch. Derm. Syph. (Chic.), 1959. 71, 506.

Marten, R. H., and Dulake, M., Brit. f. Derm., $1957,69,395$.

1953,67, 638., Arch. Derm. Syph. (Chic.).

Walters, J.' W., ibid., 1955, 72, 94.

Rynearson, E. H., Arch. interm. Hed, and 66, 851 .

\section{Colour-blindness}

Q.-Is colour blindness in children progres. sive with age, and can anything be done to prevent it getting worse?

A.-Colour blindness is a congenital anomaly, which remains stationary through out life. There is no effective treatment for the condition.

\section{Sterilization of Gloves}

Q.-How can gloves, etc., be sterilized by means of a-domestic gas cooker with "Regulo" temperature gauge? A small sterilizing drum is available about $\delta$ to 10 in. $(20$ to $25 \mathrm{~cm}$.) in diameter. Where maj some sort of marker be obtained to put into the drum or the dressings as proof of adequate sterilization?

A.-The answer is that " gloves, etc." cannot be sterilized in a domestic gas cooker. Dry heat applied at the temperature and for the time required to effect sterilization would destroy gloves and do great damage to othes items of the kind normally sterilized along with gloves for surgical operations. A reliably constructed and operated pressure steam sterilizer should be used to sterilize the loads mentioned. If sterile gloves alone are required, they may be bought presterilized by gamma radiation and in disposable form (that is, use only once, then discard). For some nonsurgical purposes gloves need not be sterilized but should be scrupulously clean and freed from any significant number of vegetative pathogenic bacteria. This can be accomplished by their being carefully and thoroughly washed on the hands of the uses in any reliable mixture of detergent and disinfectant (soap and water and a simple phenolic would suffice), rinsed in clean running water, and dried with a freshly laundered towel.

If a pressure steam sterilizer is acquired and is used to sterilize the gloves, etc., by steam, a useful and simple check on the work ing of the sterilizer may be secured by regulas use of the sterilizer control tubes manufactured by Albert Browne Ltd., Chancery Street, Leicester, England. Full details concerning the storage, use, and choice of appropriate tubes may be had from the manufacturers. These tubes are not the only such device, but they are one of the simplest and most reliable for an immediate check.

Correction.-In the issue of 19 November ( $p$ 1276) under the heading "Appointments" the degree after the name of Dr. R. J. C. Southern should have been M.R.C.P.Ed. 